\title{
PREDICTION OF TENSILE AND FRACTURE PROPERTIES OF CRACKED CARBON STEEL WIRES USING FINITE ELEMENT SIMULATION
}

\author{
Kazeem K. ADEWOLE, Steve J. BULL \\ School of Chemical Engineering and Advanced Materials, Newcastle University, \\ Newcastle upon Tyne, NE1 7RU, United Kingdom
}

Received 16 Feb 2012; accepted 18 Jan 2013

\begin{abstract}
Steel wires are used as a bridge construction material and as pre-stressing strands or tendons in pre-stressed structural units among other applications in civil engineering. To date, the estimation of the load carrying capacity of a cracked wire has been based on purely experimental classical fracture mechanics work conducted with non-standardised classical fracture mechanics specimens as standard test specimens could not be manufactured from the wire owing to their size. In this work, experimental mechanical tests and finite element simulation with the phenomenological shear fracture model has been conducted to investigate the effect of miniature cracks with dimensions less than or equal to $0.2 \mathrm{~mm}$ (which is the limit of the current non-destructive detection technology) on the tensile and fracture properties of flat carbon steel wire. The investigation revealed that the reduction in the displacement at fracture of the wire due to the presence of cracks shallower than $0.2 \mathrm{~mm}$ is significantly higher than the reduction in the fracture load of the wire. Consequently, the displacement at fracture and by extension the fracture strain capacity of the wire could serve as a more appropriate parameter to assess the quality and the structural integrity of cracked wires.
\end{abstract}

Keywords: classical facture mechanics, cracks, phenomenological shear fracture, wires, tensile properties.

Reference to this paper should be made as follows: Adewole, K. K.; Bull, S. J. 2013. Prediction of tensile and fracture properties of cracked carbon steel wires using finite element simulation, Journal of Civil Engineering and Management 20(2): 159-168. http://dx.doi.org/10.3846/13923730.2013.861862

\section{Introduction}

Steel wires are used as a bridge construction material and as pre-stressing strands or tendons in pres-stressed structural units such as beams and girders among other applications in civil engineering. Steel wire as a typical engineering material inevitably contains defects (such as scratches or cracks) arising from their manufacturing processes or through mechanical damage during transportation, construction and installation, and during the service life of the wire. These defects, particularly cracks could threaten the structural integrity of the wires, and consequently threaten the structural integrity of structures (such as suspension bridges and pre-stressed concrete structures) where wires provide the required tensile reinforcement.

The wire considered in this paper is a typical carbon steel wire used to provide tensile reinforcement for civil engineering structures, such as bridges and pre-stressed concrete structures. The manufacturers and users of this wire desire an understanding of the effect of miniature cracks with dimensions less than $0.2 \mathrm{~mm}$, which is the defect detection capability of the commercially available inline defect detection system in use in the industry on the tensile properties and fracture behavior of the wires for design and quality assurance purposes.
Mahmoud (2007) and Toribio and Valiente (2006) have recognised that the current practice used by engineers to estimate the safe load carrying capacity of cracked wires as the product of the ultimate strength and the original nominal area of the wire without taking into consideration, the fracture parameters of the wire material may overestimate the strength of the wire due to crack tip plasticity. Consequently, they recommended using a fracture mechanics based approach to estimate the safe load carrying capacity of cracked wires.

Research reported in the published literature on carbon steel wires, such as that conducted by Mahmoud (2007) on cracked bridge cable wires, Toribio and Valiente (2004, 2006) on cracked concrete pre-stressing wires have focused on estimating the fracture strength of cracked wires. The work of these authors was based on the classical fracture mechanics approaches using the linear elastic fracture mechanics (LEFM) and the net section theory/plastic collapse fracture mechanics. The laboratory tests conducted by these researchers used non-standardised classical fracture mechanics specimens (i.e. different researchers used different specimen sizes) as standard test specimens could not be manufactured from the wire owing to their size. When standard fracture mechanics specimens cannot be obtained and when a safe use of the classical fracture mechanics concepts cannot be ensured, micromechanism- 
based fracture mechanics models serve as an alternative methodology for investigating the behaviour of cracked structures and components (Pardoen et al. 2010). Phenomenological models are one of the micromechanism-based fracture mechanics models that predict ductile fracture based on the assumption that ductile fracture occurs when a weighted measure of the accumulated plastic strain, such as the equivalent plastic strain reaches a critical value (Dunand, Mohr 2009).

The work in this paper was undertaken to provide an understanding of the effect of miniature cracks on the tensile properties of flat carbon steel wire and to provide the technical data on the crack size acceptance criteria to be incorporated into the wire's standard for the quality control and quality assurance of the wires. The design charts presented in this paper could also be used to estimate the tensile properties of cracked wires for the structural integrity or engineering critical assessment of the wire needed for the fitness for purpose assessment of civil engineering structures where wires are used to provide tensile reinforcement. The laboratory and finite element (FE) tensile testing of the crack-free and notched precracked wire specimens conducted with the isotropic elastic-plastic model combined with the phenomenological shear failure criterion inbuilt in the Abaqus 6.9-1finite element (FE) code is presented. The investigation covered the effect of crack depths from $0.2 \mathrm{~mm}$ to $0.05 \mathrm{~mm}$ on the yield load, the ultimate load, the fracture load and the displacement at fracture of wires with $12 \times 5 \mathrm{~mm}$ and $12 \times 7 \mathrm{~mm}$ cross-sectional dimensions.

\section{Isotropic elastic-plasticity model}

The isotropic elastic-plasticity model in Abaqus is based on a linear isotropic elasticity theory and a uniaxial-stress, plastic-strain strain-rate relationship (Simulia 2007). The elastic aspect of the model is defined in terms of its volumetric and deviatoric components given in Eqns (1) and (2), respectively, obtained from Simulia (2007). The model is based on a von Mises yield surface with the yield function, $f$, given in Eqn (3) and a flow rule given in Eqn (4) obtained from Simulia (2007):

$$
\begin{gathered}
p=-K \varepsilon_{v o l} ; \\
S=2 G e^{e l} ; \\
f=q=\sqrt{\frac{3}{2}} S: S ; \\
d e^{p l}=d e^{p l} n,
\end{gathered}
$$

where: $p$ is the hydrostatic stress; $\varepsilon_{v o l}$ is the volume strain; $S$ is the deviatoric stress; $e^{e l}$ is the deviatoric elastic strain; $q$ is the von Mises equivalent stress; $e^{p l}$ is the deviatoric plastic strain; $\bar{e}^{p l}$ is the equivalent plastic strain; $n=\frac{3}{2} \frac{S}{q} ; K$ is the bulk modulus; $G$ is the shear modulus. $K$ and $G$ are calculated from the Young's modulus $E$, and Poisson's ratio $v$ of the material.
The isotropic elastic-plastic modelling parameters are the: density, Poisson's ratio, Young's modulus and the post yield true stress and true plastic strain. Details of the isotropic elastic-plastic modelling parameters employed for the FE tensile testing of the wires considered in this work are presented in Section 1.2.

\section{Shear failure criterion}

The shear failure criterion is a phenomenological model for predicting the onset of damage due to shear bands. Applied stress causes shear band formation and localisation, leading to the formation of cracks within the shear bands and eventual failure (Simulia 2007). The shear model assumes that the equivalent plastic strain at the onset of damage $\bar{\varepsilon}_{s}^{p l}$ is a function of the shear stress ratio $\theta_{s}$ and equivalent strain rate $\dot{\bar{\varepsilon}}^{p l}$. The shear stress ratio is calculated using the expression in Eqn (5) obtained from Simulia (2007):

$$
\theta_{S}=\left(q+k_{s} p\right) / \tau_{\max },
$$

where: $\tau_{\max }$ is maximum shear stress; $k_{S}$ is material parameter.

The criterion for shear damage initiation is met when the condition in Eqn (6) is satisfied:

$$
\omega_{S}=\int \frac{d \bar{\varepsilon}^{p l}}{\bar{\varepsilon}_{S}^{p l}\left(\theta_{S}, \dot{\bar{\varepsilon}}^{p l}\right)}=1,
$$

where $\omega_{S}$ is a state variable, that increases monotonically with plastic deformation proportional to the incremental change in equivalent plastic strain. At each increment during the analysis the incremental increase in $\omega_{S}$ is calculated using the expression in Eqn (7) obtained from Simulia (2007):

$$
\Delta \omega_{S}=\frac{\Delta \bar{\varepsilon}^{p l}}{\bar{\varepsilon}_{S}^{p l}\left(\theta_{S}, \dot{\bar{\varepsilon}}^{p l}\right)} \geq 0 .
$$

\section{Calibration of shear failure modelling parameters}

The parameters needed for phenomenological shear failure modelling can be obtained experimentally. However, obtaining these parameters through direct experimentation may be difficult because it would require experiments over a range of shear stress ratio (Simulia 2007). The phenomenological shear failure modelling parameters can also be obtained from Hooputra et al. (2004) simplified analytical expression given in Eqn (8). Similarly, obtaining the equivalent plastic strain at the onset of shear damage, $\bar{\varepsilon}_{s}^{p l}$, from Hooputra et al. (2004) simplified analytical expression also requires a number of specially designed experiments (interested readers are referred to Hooputra et al. (2004) for details of the specially designed experiments) to determine four parameters: $k_{S}, \varepsilon_{S}^{+}, \varepsilon_{\bar{S}}$ and $f$ (Simulia 2007; Hooputra et al. 2004). In addition, the simplified analytical expression may give very high values of the equivalent plastic strain at damage initiation when the shear stress ratio is very small (Simulia 2007): 


$$
\bar{\varepsilon}_{s}^{p l}=\frac{\varepsilon_{S}^{+} \sinh \left[f\left(\theta^{+}-\theta^{-}\right)\right]+\varepsilon_{S}^{-} \sinh \left[f\left(\theta^{+}-\theta^{-}\right)\right]}{\sinh \left[f\left(\theta^{+}-\theta^{-}\right)\right]},
$$

where: $\theta^{+}$and $\theta^{-}$are the values of the parameter $\theta$ for equibiaxial tension and compression; $\varepsilon_{S}^{+}$and $\varepsilon_{S}^{-}$are the equivalent plastic strain in equibiaxial tension/ compression at shear fracture and $f$ is an orientation dependent parameter.

Considering the scope and scale of the experiments required to obtain the shear failure modelling parameters from direct experimentation or from Hooputra et al. (2004) simplified analytical expression, and the limitation of Hooputra et al. (2004) simplified analytical expressions, the determination of the phenomenological fracture modelling parameters remains predominantly a phenomenological fitting procedure which requires a combination of testing and numerical simulations (Bernauer, Brocks 2002). The phenomenological fitting procedure involves keeping some parameters constant and varying others during numerical simulations until the simulation results fit the experimental data usually up to the fracture initiation point which is marked by a sudden drop of load in tension tests. The values of the set of damage and fracture modelling parameters at which the numerical data fits with the experimental data are generally taken as the calibrated or critical fracture parameters (Bernauer, Brocks 2002). Details of the phenomenological fitting procedure employed to obtain the calibrated shear damage and fracture modelling parameters for the wires considered in this work are presented in Section 1.2.

\section{Experiment}

Details of the laboratory and numerical experimental methodologies are presented in this section.

\subsection{Laboratory tensile testing}

Laboratory tensile tests were conducted on $50 \mathrm{~mm}$ gauge length crack-free and notched pre-cracked (with $2 \mathrm{~mm}$ deep, 60 degree V-notch with a notch tip/root radius of $0.25 \mathrm{~mm}$ and $1 \mathrm{~mm}$ crack) wire specimens shown in Figures 1(a) and (b), respectively. The wire specimens were pre-cracked with an Instron 8511 testing machine by subjecting the notched wire specimen to 33,450 cycles of axial loading conducted at $0.13 \%$ to $18.47 \%$ of the ultimate tensile load of the wire. The laboratory tensile testing was conducted with an Instron IX4505 universal testing machine and the displacement was measured using an Instron 2630-112 clip-on strain gauge extensometer with a $50 \mathrm{~mm}$ gauge length.

\subsection{FE tensile testing simulation}

The isotropic elastic-plastic modelling parameters employed for the simulation of the undamaged tensile behaviour of the wire specimens are the density of $7.6 \times 10^{-6} \mathrm{~kg} / \mathrm{mm}^{3}$ and Poisson's ratio of 0.3 which are typical values for steel obtained from Simulia (2007). Others are the Young's modulus of $200 \times 10^{3} \mathrm{~N} / \mathrm{mm}^{2}$, and the post yield true stress and true plastic strain up to the true ultimate tensile stress and its associated true plastic strain obtained from the engineering stress and strain of the wires, which were obtained from the wires' laboratory tensile test force-displacement results. For confidentiality (non-disclosure agreement on the tensile properties of the wires), the values of the yield and ultimate tensile stresses and their associated strains, and the ultimate load and displacement at fracture of the wires are not presented in this paper.

The calibrated shear damage and fracture modelling parameters employed for the simulation of the tensile testing of the wire specimens were obtained through the phenomenological curve fitting procedure. For both wire sizes considered in this work, tensile testing simulations were conducted on crack-free wire specimens shown in Figure 2(a) with varying shear damage and fracture modelling parameter combinations until the FE predicted a force-displacement curve that agreed with the experimental curve and with the same fracture initiation point as the experimental curve. A few of the parameter combinations designated as parameter combinations $\mathrm{A}, \mathrm{B}, \mathrm{C}$ and $\mathrm{D}$ considered for the $12 \times 5 \mathrm{~mm}$ wire specimens are presented in the Table. Parameter combination A which was the starting parameter values are typical parameters for ductile materials obtained from Simulia (2007). The modelling parameters combination at which the FE simulation predicted a force-displacement curve that agreed with the experimental curve and with the same fracture initiation point as the experimental curve was taken as the calibrated shear damage and fracture modelling parameters for the wires.

Three dimensional (3D) FE simulation of the tensile testing of the crack-free wire specimen was conducted on the simplified/idealised wire specimen model shown in Figure 2(a) with the filleted corners in the actual wire shape in Figure 1(a) idealised as sharp corners in the FE model to simplify the FE analysis and keep model size reasonable as recommended by Simulia (2007). The simplified model was meshed with global $1 \times 1 \times 1 \mathrm{~mm} \mathrm{C3D8R}$ elements (8-node hexahedral linear brick reduced integration elements with hourglass control) as shown in Figure 2(a). The local mesh at the middle of the three dimensional model was meshed with finer elements with $1 \times 0.25 \times 0.25 \mathrm{~mm}$ (length $\times$ width $\times$ thickness) dimension as shown in Figure 2(a) to obtain the cup and cone fracture exhibited by the experimental test specimens as shown later in Section 2. The left hand end of the model was fixed and the right hand end, which is free to move only in the direction of the tensile load, was subjected to a longitudinal displacement as shown in Figure 2(a).

As shown later in Figure 3, only the simulation conducted with the parameters combination $\mathrm{B}$ predicted a force-displacement curve with approximately the same fracture initiation point as the experimental curve. As discussed later in Section 3, the values of the modelling parameters in parameters combination B thus serve as the calibrated shear damage and fracture parameters. Consequently, subsequent simulations conducted to study the effects of cracks on the wires were conducted with the shear failure modelling parameters combination $\mathrm{B}$. 


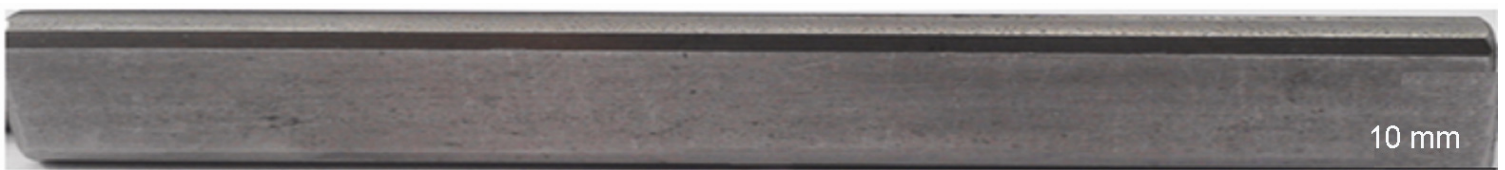

a) Crack-free wire specimen

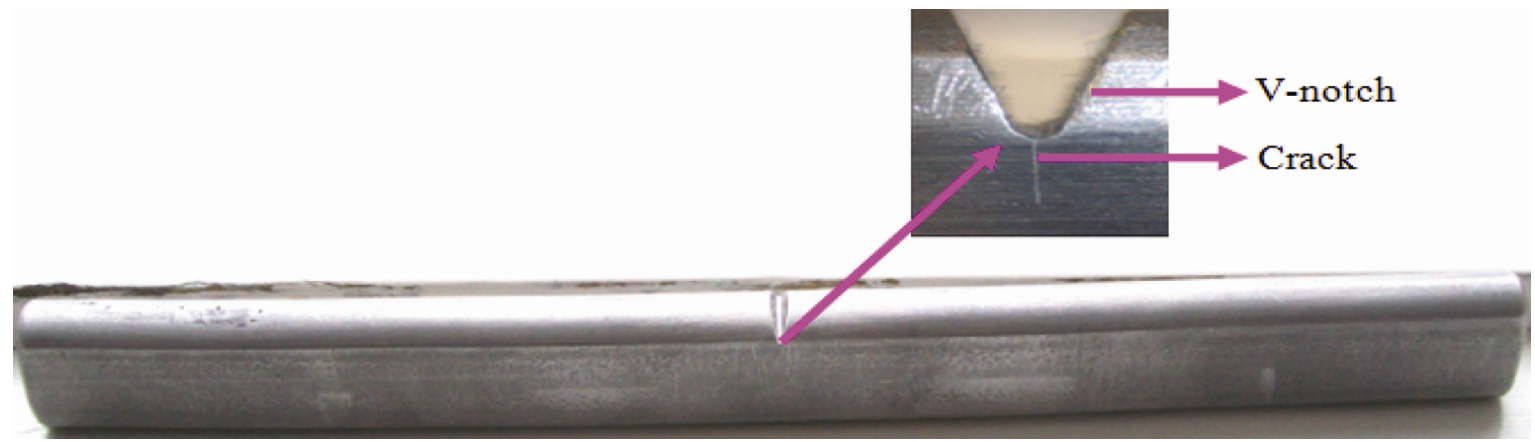

b) Notched and pre- cracked wire specimen

Fig. 1. Crack-free, and notched pre-cracked laboratory wire specimens

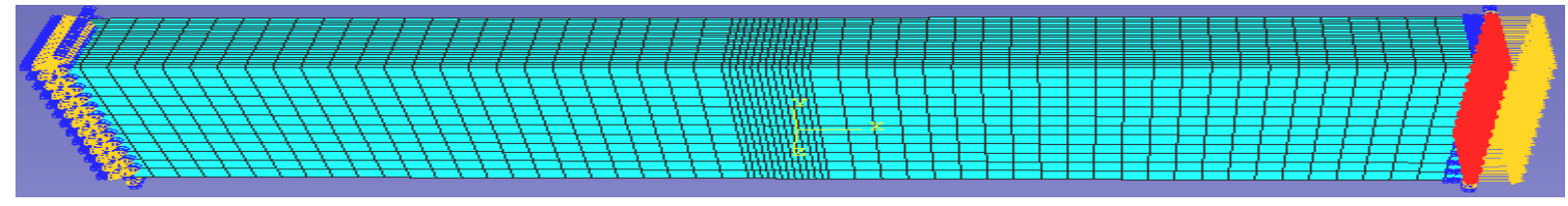

a) 3D Model of the crack-free wire specimen

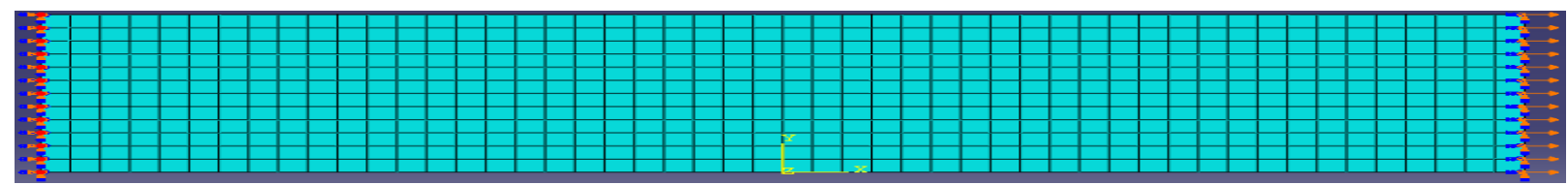

b) 2D Model of the crack-free wire specimen

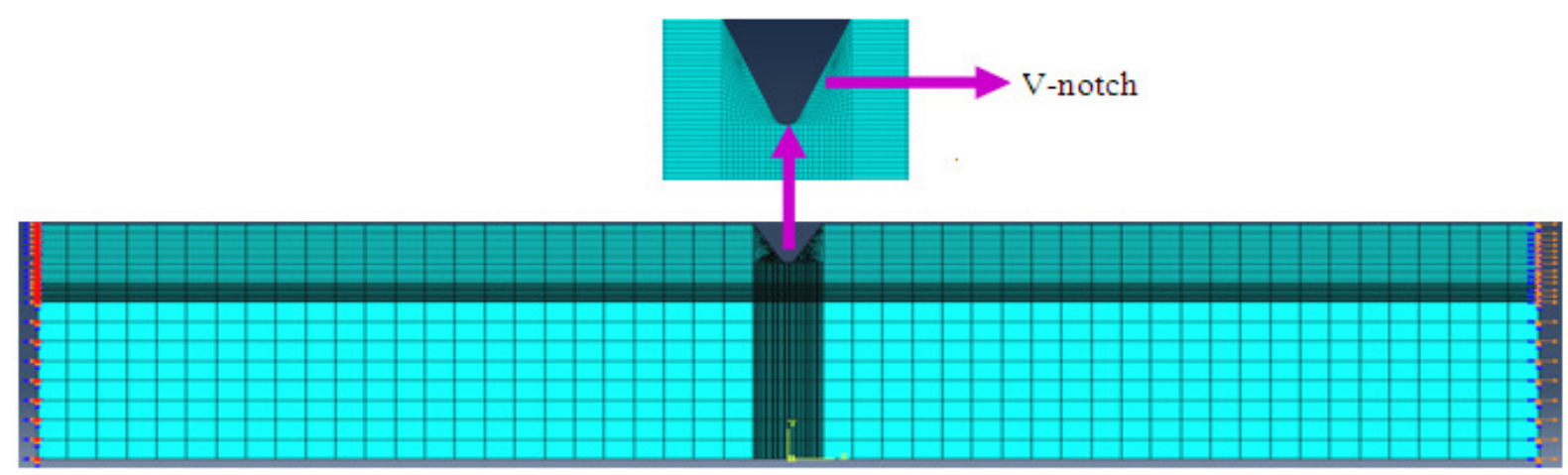

c) Meshed model of notched pre-cracked wire specimen

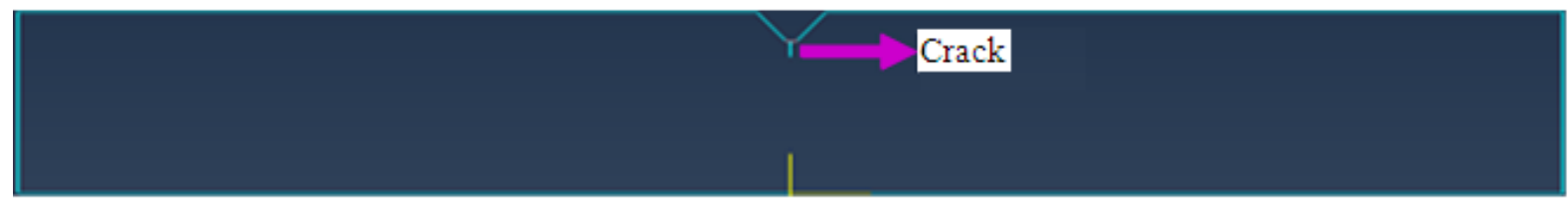

d) Model of notched pre-cracked wire specimen in wire frame

Fig. 2. FE models of crack-free and notched pre-cracked wire specimens 
Table. Shear fracture parameter combinations investigated

\begin{tabular}{c|c|c|c|c|c}
\hline $\begin{array}{c}\text { Parameters } \\
\text { combinations }\end{array}$ & $\begin{array}{c}\text { Equivalent plastic strain at } \\
\text { onset of shear damage }\end{array}$ & $\begin{array}{c}\text { Shear stress } \\
\text { ratio }\end{array}$ & $\begin{array}{c}\text { Strain rate } \\
\left(\mathrm{s}^{-1}\right)\end{array}$ & $\begin{array}{c}\text { Parameter, } \\
\text { Ks }\end{array}$ & $\begin{array}{c}\text { Plastic displacement } \\
\text { at failure }\end{array}$ \\
\hline A & 0.2761 & 10.0 & 0.0001 & 0.3 & 0.1 \\
\hline B & 0.3451 & 12.5 & 0.000125 & 0.2 & 0.2 \\
\hline C & 0.4142 & 15.0 & 0.00015 & 0.4 & 0.3 \\
\hline D & 0.5522 & 20.0 & 0.0002 & 0.1 & 0.4 \\
\hline
\end{tabular}

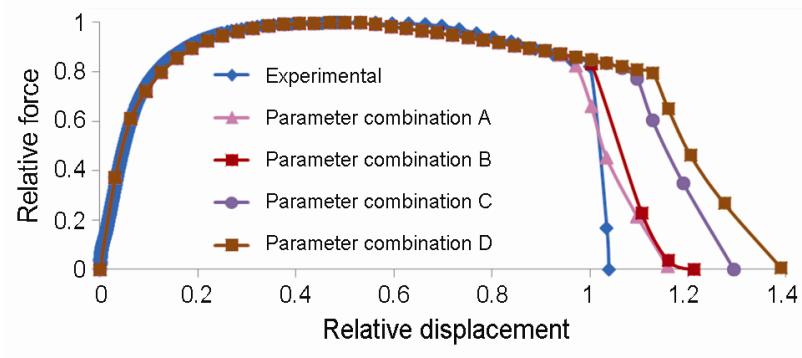

Fig. 3. Force-displacement curves from simulations with modelling parameter combinations A to D

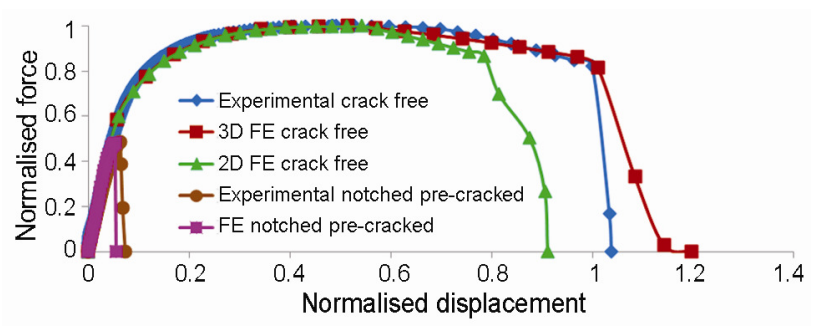

Fig. 4. Experimental and FE force-displacement curves for crack-free and notched pre-cracked wire specimens

The 3D FE simulation of the tensile testing of the cracked wire specimen could not be conducted due to the large computation time and computer resources needed for $3 \mathrm{D}$ simulation with very fine mesh around the crack tip. The mesh refinement around the crack tip is required for accurate FE simulation. Consequently two dimensional simulations were also conducted.

Figures 2(b) and 2(c) show the 2D models of the crack-free wire and the notched pre-cracked wire specimens with the same boundary conditions as the model used for the three dimensional simulations. The crack was modeled as a seam, which is used in Abaqus finite element code to model cracks and faces that are originally closed but open during analyses. The crack is therefore not visible in the meshed model shown in Figure 2(c) and is thus presented in the wireframe image of the model in Figure 2(d). The crack tip was meshed with $0.02 \times 0.02 \mathrm{~mm}$ elements as shown in Figure 2(c), which was established through mesh convergence tests to be the optimum mesh size for accurate simulation. No mesh refinement was needed for the two dimensional model of the crack-free wire to predict the cup and cone fracture as shown later in Figure 5(c) in section 2 and the model was meshed with the global $1 \times 1 \mathrm{~mm}$ elements as shown in Figure 2(b). The simulations of the tensile testing of the crack-free and notched pre-cracked wire specimens were validated and FE simulation was subsequently used as the virtual experiment to investigate the effect of miniature crack depths on the tensile and fracture properties of the wire.

\section{Results}

For confidentiality, the unit of the von Mises stress in this paper is not stated and the force-displacement curves are normalised with the experimental ultimate load and the experimental displacement at fracture. The normalised experimental force-displacement curve and the forcedisplacement curves predicted by the simulations conducted with the shear failure modelling parameter combinations $A$ to $D$ for the $12 \times 5 \mathrm{~mm}$ wire are shown in Figure 3. The normalised experimental curves and the normalized force-displacement curves predicted by the 2D and 3D FE simulations of the tensile testing of the crack-free and the notched pre-cracked wire specimens are shown in Figure 4.

The fractured wire specimens obtained from the laboratory tensile testing and from the 3D and 2D FE tensile testing of the crack-free wire specimens are shown in Figures 5 (a), (b) and (c), respectively. The experimental and 2D FE predicted fracture shapes for the notched precracked wire specimens are shown in Figures 6(a) and (b), respectively. The necked 2D wire specimen model showing the lateral (along width) contraction alone and the necked 3D wire specimen model showing the lateral and transverse contractions of the necked regions of the specimens are shown in Figures 7(a) and (b), respectively. In all the FE predicted deformed and fracture shapes, the highest and the lowest von-Mises equivalent stresses are indicated at the top and bottom of the contour plots by the deepest red colour and the deepest blue colour, respectively.

The assembled fractured experimental crack-free and notched pre-cracked wire specimens showing the reduction in thickness and width of the necked region are shown in Figures 8(a) and (b), respectively.

The typical variation of the force-displacement curves obtained from the simulation of the tensile testing of the $12 \times 5 \mathrm{~mm}$ wire with crack depth is shown in Figure 9. A Similar variation in the force-displacement response with crack depth was obtained for the $12 \times 7 \mathrm{~mm}$ wire. The typical variations of the yield load, ultimate load, fracture load and the displacement at fracture with crack depth for the $12 \times 5 \mathrm{~mm}$ wire are as shown in Figures 10-13, respectively. Similar variations of the yield load, ultimate load, fracture load and the displacement at fracture with crack depths were obtained for the $12 \times 7 \mathrm{~mm}$ wire. 

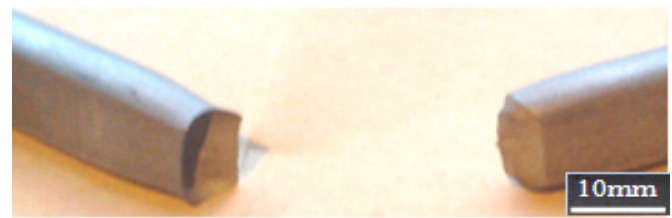

a) Fractured experimental wire specimen
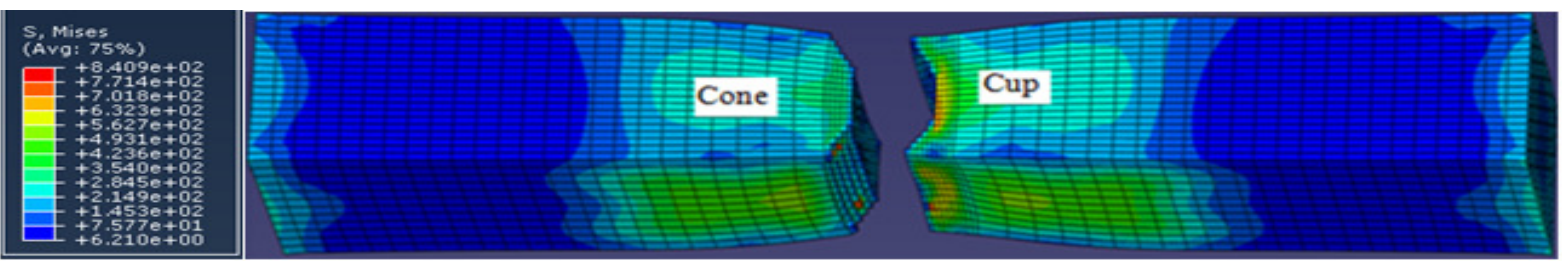

b) 3D FE predicted fractured wire specimen

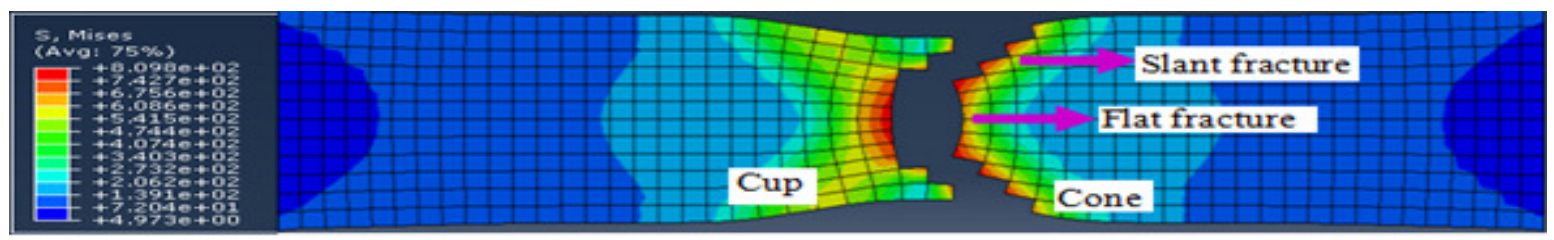

c) 2D FE predicted fractured wire specimen

Fig. 5. Experimental and FE predicted fracture shapes for crack-free wire specimens
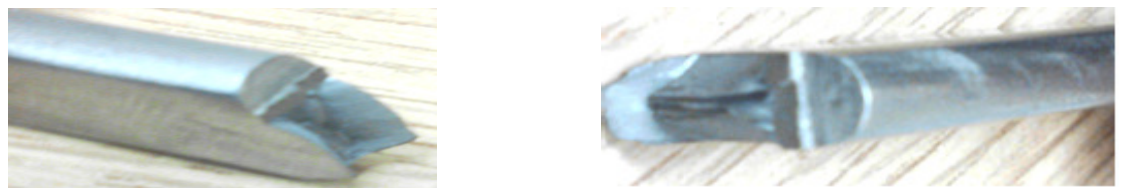

a) Fractured experimental wire specimen

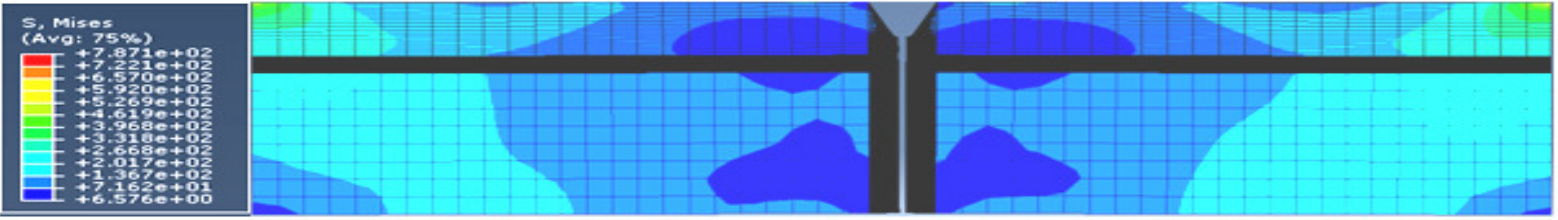

b) 2D FE predicted fractured wire specimen

Fig. 6. Experimental and FE predicted fracture shapes for notched pre-cracked wire specimens

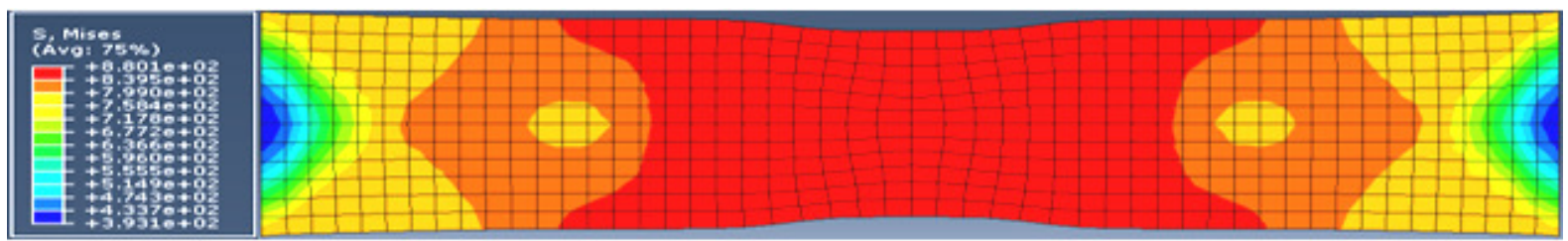

a) Lateral contraction alone in 2D simulation

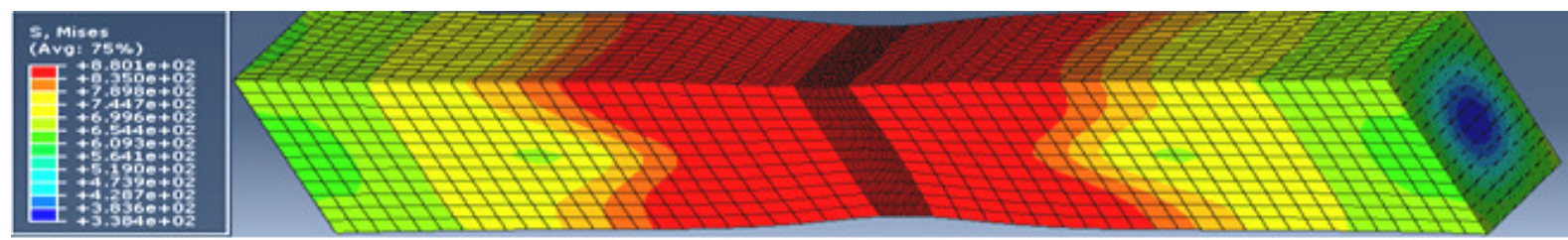

b) Lateral and transverse contractions in 3D simulation

Fig. 7. Necking of FE wire models 


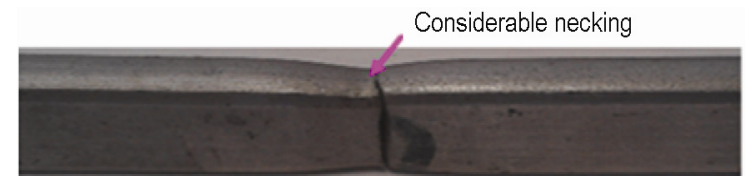

a) Assembled fractured crack-free wire specimen pieces

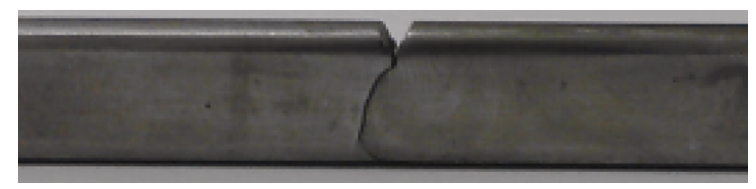

b) Assembled fractured notched pre-cracked wire specimen pieces

Fig. 8. Necking of experimental specimens

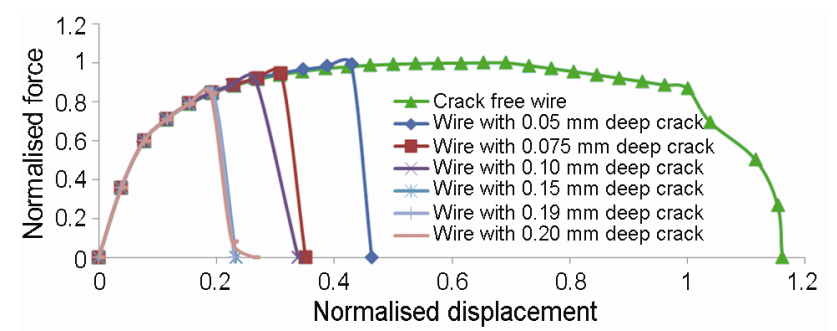

Fig. 9. Variation of stress-strain response of $12 \times 5 \mathrm{~mm}$ wire with crack depths

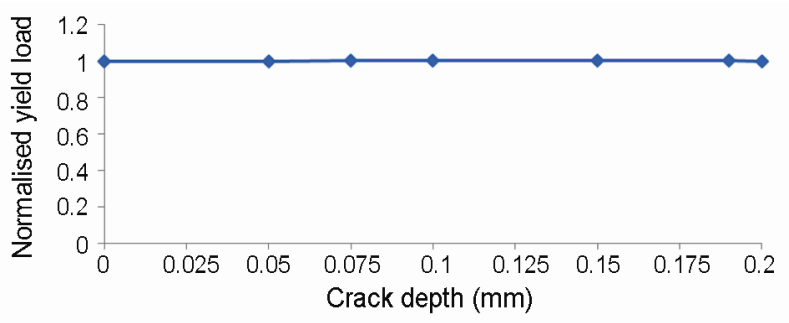

Fig. 10. Variation of yield load of $12 \times 5 \mathrm{~mm}$ wire with crack depth

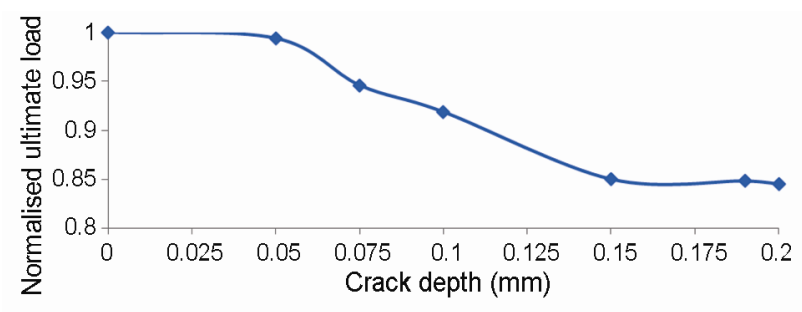

Fig. 11. Variation of ultimate load of $12 \times 5 \mathrm{~mm}$ wire with crack depth

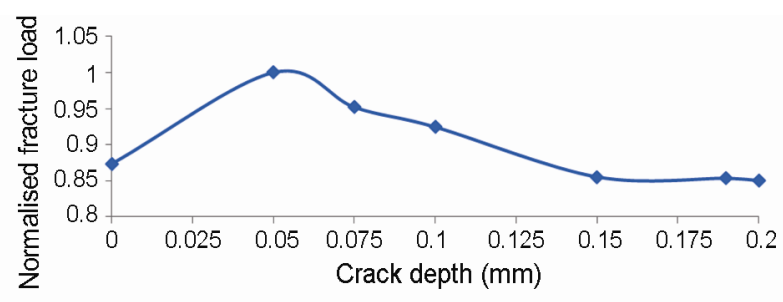

Fig. 12. Variation of fracture load of $12 \times 5 \mathrm{~mm}$ wire with crack depth

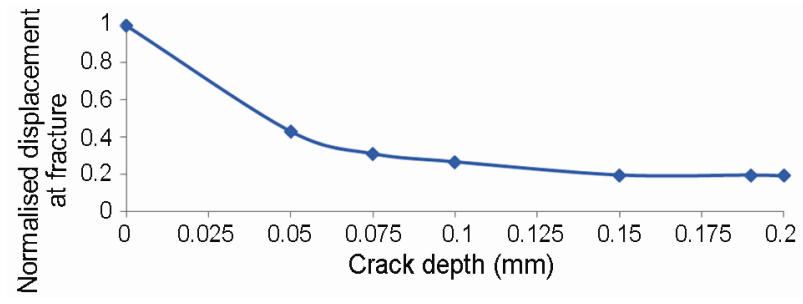

Fig. 13. Variation of displacement at fracture of $12 \times 5 \mathrm{~mm}$ wire with crack depth

\section{Discussion}

As shown in the 2D and 3D FE predicted deformed shapes of the wire specimens in Figures 7(a) and (b), respectively, the necked region at the middle of the specimen is the most stressed region of the specimen with the maximum von Mises equivalent stress of 880.1. Figures 5(b) and (c) show that the necked region of the fractured $3 \mathrm{D}$ and $2 \mathrm{D}$ wire specimen models around the fracture surface has the highest residual Mises stress of 840.9 or 809.8 that are less than the maximum Mises stress of 880.1 in the necked specimen model shown in Figures 7(a) and (b) due to stress relief at the fracture surfaces. The magnitude of the residual stress reduces along the length of the fractured wire specimen model from the maximum value at the fracture surface as shown by the deep red to light green colour shade in the necked region and the light blue to the deep blue in the un-necked regions (with the exception of the two ends of the specimen with relatively high residual stresses due to the effect of the boundary conditions at the two ends) with the least residual stress. This result is expected considering the fact that the necked and the unnecked regions are the most and the least stressed regions of the wire specimen models.

On the basis of a good agreement between the experimental and all the FE predicted force-displacement curves shown in Figure 3, all the simulations conducted with the shear failure modelling parameter combinations A to $\mathrm{D}$ are able to predict the tensile response of the crack-free wire well beyond the ultimate load point. However, only the force-displacement curve predicted by the simulation conducted with parameters combination B accurately predicted the same fracture initiation point as the experimental curve. Thus, it can be concluded that the values of the shear failure modelling parameters in parameters combination $\mathrm{B}$ serve as the calibrated shear damage and fracture parameters for the wire, and the tensile properties (yield load, ultimate tensile load and displacement at fracture) predicted by the simulation conducted with the parameters combination B represent the tensile properties of the crack-free wire.

As shown in Figure 4, there is a good agreement between the force-displacement curves obtained from the laboratory, 2D and 3D FE tensile testing of the crack-free wire specimen up to the ultimate load point of the wire, and a good agreement between force-displacement curves obtained from the laboratory and 2D FE tensile testing of the notched pre-cracked wire specimens. The lower displacement values from the displacement at the ultimate load point to the displacement at fracture predicted by the 
2D simulation of the tensile testing of crack-free specimen agree with what is reported by Cabezas and Celentano (2004) and are due to the fact that the 2D simulation merely captured the longitudinal extension resulting from the lateral contraction alone as shown in Figure 7(a). The 2D simulation did not capture the longitudinal axial elongation which accompanied the transverse contraction of the specimen during necking as shown in the necked 3D FE specimen and the assembled experimental specimen shown in Figures 7(b) and 8(a). However, the 2D simulation of the tensile testing of the notched pre-cracked specimen predicted the same displacement at fracture as the experimental notched precracked wire specimen, which did not exhibit any significant necking as shown in Figure 8(b). This result indicates that the $2 \mathrm{D}$ simulation provides sufficiently accurate prediction of the tensile and fracture properties of the cracked wire in the absence of necking as for the precracked wires tested here.

Also there is a good agreement between the "cup and cone" fracture exhibited by the fractured experimental crack-free specimens shown in Figure 5(a) and the "cup and cone" fracture predicted by $3 \mathrm{D}$ and $2 \mathrm{D}$ FE tensile testing of the crack-free wire as shown in Figures 5(b) and (c). The cup and cone fracture is characterized by a flat or normal fracture in the center of the specimen and a slant fracture at the outer regions of the specimen (Scheider, Brocks 2003; Anderson 2005). The good agreements between the experimental and FE forcedisplacement curves and the "cup and cone fracture" exhibited by the fractured experimental specimen and the FE model confirm the accuracy of the FE simulation. The good agreements also shows that the shear phenomenological fracture model, which has been identified by Adewole et al. (2011) as an appropriate fracture model for the prediction of the fracture behavior of the crackfree wire considered in this work is suitable for the prediction of the tensile and fracture behaviours of the cracked wire.

The presence of cracks with depth from 0.05 to $0.19 \mathrm{~mm}$ has no significant effect on the yield load and the displacement at which the wire yields as shown in Figures 9 and 10 with only $0.38 \%$ and $0.30 \%$ maximum percentage reduction in the yield load at $0.19 \mathrm{~mm}$ crack depth for the $12 \times 5 \mathrm{~mm}$ and $12 \times 7 \mathrm{~mm}$ wires respectively. As shown in Figure 9, only the force-displacement curve from the crack-free wire specimen has a distinct ultimate load point and distinct fracture point. For all the cracked wire specimens, the ultimate load point serves as the fracture point. This shows that the presence of cracks in the wire changed the tensile and fracture behaviours of the wire, causing the wire to fracture at the ultimate load of the cracked wire without necking (since necking begins at the ultimate load) as shown in Figure 8(b), compared with the significant necking exhibited by the crackfree wire specimen shown in Figures 7 and 8(a). Thus the presence of cracks reduces the ductility of the wire and embrittles the wire. The embrittlement or loss in the ductility of the wire could be attributed to the high local stress and strain concentrations at the crack tip, the high local strain rate around the notches and the high local strain hardening at the crack tip, all of which promote cracking, crack extension, and brittle fracture as stated by Dieter (1998).

As shown in Figures 11 and 12, the presence of cracks generally reduces the ultimate load and the fracture load of the wire and the reduction is proportional to the crack depth. In Figure 12, the fracture loads of the cracked wire specimens with crack depth up to approximately $0.125 \mathrm{~mm}$ are higher than the fracture load of the crack-free wire because as shown in Figure 9, beyond the ultimate load point, the load sustained by the wire is inversely proportional to the displacement. This explains why the crack-free wire with a higher ductility (with higher quality) as typified by the higher displacement at fracture has lower fracture load than the cracked wire with reduced ductility. Consequently the fracture load and by extension the fracture stress commonly used to characterize the quality and access the structural integrity of cracked wires may not be the best indicator of the quality and the structural integrity of the cracked wire and the displacement at fracture and by extension, the fracture strain could serve as a better indicator of the quality and structural integrity of cracked wire.

The reduction in the ultimate load and/or the fracture load of the wire could be attributed to the reduction in the gross cross-sectional area of the wire and consequently the reduction in the global load carrying capacity of the wire. The reduction could also be attributed to the fact that the high local strain hardening around the tip of the crack embrittles the material around the crack tip thereby promoting crack propagation in the wire at a lower applied load. For the $12 \times 5 \mathrm{~mm}$ wire, the presence of cracks with depth from 0.05 to $0.19 \mathrm{~mm}$ reduced the displacement at fracture and the ultimate load (and invariably the fracture load of the wire, since the ultimate load and the fracture load of the cracked wire are the same) by $57.07 \%$ to $81.00 \%$ and $0.62 \%$ to $15.17 \%$, respectively. Similarly, for the $12 \times 7 \mathrm{~mm}$ wire, the presence of cracks with depth from 0.05 to $0.19 \mathrm{~mm}$ reduced the displacement at fracture and the ultimate load and/or the fracture load of the wire by $59.36 \%$ to $84.97 \%$ and $2.39 \%$ to $15.98 \%$, respectively.

The $81.00 \%$ reduction in the displacement at fracture of the $12 \times 5 \mathrm{~mm}$ wire by $0.19 \mathrm{~mm}$ deep crack is significantly higher than the $15.17 \%$ reduction in the fracture load of the wire by the same crack depth. Similarly, the $84.97 \%$ reduction in the displacement at fracture of the $12 \times 7 \mathrm{~mm}$ wire by $0.19 \mathrm{~mm}$ deep crack is significantly higher than the $15.98 \%$ reduction in the fracture load of the wire by the same crack depth. These results indicate that the presence of a crack has a more detrimental effect on the displacement at fracture and consequently on the ductility of the wire, which further stresses the need to use the displacement at fracture or by extension the fracture strain of the wire rather than the fracture load or the fracture strain as the indicator of the quality and structural integrity of cracked wires

The charts in Figures 11, 12 and 13 which show the relationship between the crack depth and the ultimate 
load, the fracture load and the displacement at fracture of the wire could be used as the basis of design charts to determine the maximum crack size that can be accepted for a target ultimate load, fracture load and displacement at fracture. More work on the variability of wire properties and on the properties of other wire steels would be needed to develop this further. Furthermore, the design chart approach could be extended to determine the ultimate load, fracture load and displacement at fracture of cracked wire which could be used for engineering critical assessment of the wire in service needed for the fitness for purpose assessment of bridges, pre-stressed concrete structures and other civil engineering structures where wires provide tensile reinforcement.

\section{Conclusions}

In this paper, the experimental and simulation techniques employed to predict the tensile and fracture behaviour of flat carbon steel wire are presented. It is demonstrated that both 2D and 3D FE simulations with the phenomenological shear fracture model are able to predict the tensile and fracture behaviour of crack-free and notched precracked wires based on the predicted force-displacement response and the "cup and cone" fracture shape which agree well with the properties exhibited by experimental wire specimens. Thus, it is established that a simple tensile test and tensile testing simulation with the micromechanical-based shear phenomenological fracture model can be used to predict the fracture load and other tensile and fracture behaviour/properties of cracked wires. Consequently, the use of the micromechanical-based shear phenomenological fracture model to predict the fracture load and other tensile and fracture behaviour/properties of cracked wires serves as a suitable alternative to using non-standardised classical fracture mechanics specimens with various researchers using various non-standardised specimen sizes as presented in the published literature to predict the fracture properties of wires. The use of the micromechanical-based shear phenomenological fracture model to predict the fracture load and other tensile and fracture behaviour/properties of cracked wires also serves as an alternative to using the current practice used by engineers to estimate the safe load carrying capacity of cracked wires as the product of the ultimate strength and the original nominal area of the wire which does not take into consideration the fracture parameters of the wire.

FE tensile testing simulation was employed as a virtual experiment to investigate the effect of miniature crack with depth from $0.05 \mathrm{~mm}$ to $0.19 \mathrm{~mm}$. The investigation revealed that the presence of miniature cracks shallower than $0.19 \mathrm{~mm}$ embrittles the wire as evidenced in the substantial reduction in the displacement at fracture of the wire. The investigation also revealed that the presence of miniature cracks has a more detrimental effect on the displacement at fracture and consequently on the ductility of the wire than on the fracture load of the wire. This result indicates the need to use the displacement at fracture (by extension the fracture strain) of the wire rather than the fracture load (by extension the fracture stress) of the wire for the quality assessment and the structural integrity of the cracked wires.

Also presented in this paper are the design charts showing the relationships between the crack depth and the ultimate load, the fracture load and the displacement at fracture of the flat carbon steel wire considered in this research. These design charts could be used to estimate the mechanical parameters of cracked wires for the structural integrity assessment of the wire and for the fitness for purpose assessment of flexible pipes and other civil engineering structures where wires are used to provide tensile reinforcement. The design charts could also be used to determine the maximum crack size that can be accepted for a desired ultimate load, fracture load and displacement at fracture, and thus provides the technical data required to specify the limit of acceptable crack size in the flat wire standards for the quality control and quality assurance purposes.

\section{References}

Adewole, K. K.; Julia, M. R.; Bull, S. J. 2011. Determination of the appropriate fracture mechanism for flexible pipe tensile armour wires using the micro-mechanism based fracture mechanics, in Ninth International Conference on Advances and Trends in Engineering Materials and their Applications (AES-ATEMA'2011), 1-5 August, 2011, Montreal, Canada, 147-154.

Anderson, T. L. 2005. Fracture mechanics fundamentals and applications. $3^{\text {rd }}$ ed. CRC press, Taylor and Francis Group. 640 p.

Bernauer, G.; Brocks, W. 2002. Micro-mechanical modelling of ductile damage and tearing-results of a European numerical round robin, Fatigue \& Fracture of Engineering Materials \& Structures 25(4): 363-384. http://dx.doi.org/10.1046/j.1460-2695.2002.00468.x

Cabezas, E. E.; Celentano, D. J. 2004. Experimental and numerical analysis of the tensile test using sheet specimens, Finite Elements in Analysis and Design 40(5-6): 555575. http://dx.doi.org/10.1016/S0168-874X(03)00096-9

Dieter, G. E. 1998. Mechanical metallurgy. McGraw-Hill Book Company. $659 \mathrm{p}$.

Dunand, M.; Mohr, D. 2010. Hybrid experimental-numerical analysis of basic ductile fracture experiments for sheet metals, International Journal of Solids and Structures 47(9): 1130-1143. http://dx.doi.org/10.1016/j.ijsolstr.2009.12.011

Hooputra, H.; Gese, H.; Dell, H.; Werner, H. A. 2004. Comprehensive failure model for crashworthiness simulation of aluminium extrusions, International Journal of Crashworthiness 9(5): 449-464. http://dx.doi.org/10.1533/ijcr.2004.0289

Mahmoud, K. M. 2007. Fracture strength for a high strength steel bridge cable wire with a surface crack, Theoretical and Applied Fracture Mechanics 48(2): 152-160. http://dx.doi.org/10.1016/j.tafmec.2007.05.006

Pardoen, T.; Scheyvaertsa, F.; Simara, A.; Tekoglu, C.; Onck, P. R. 2010. Multiscale modeling of ductile failure in metallic alloys, Comptes Rendus Physique 11(3-4): 326-345. http://dx.doi.org/10.1016/j.crhy.2010.07.012

Scheider, I.; Brocks, W. 2003. Simulation of cup-cone fracture using the cohesive model, Engineering Fracture Mechanics 70: 1943-1961. http://dx.doi.org/10.1016/S0013-7944(03)00133-4 
Simulia. 2007. Abaqus documentation. Abaqus Incorporation, Dassault Systemes.

Toribio, J.; Valiente, A. 2004. Approximate evaluation of directional toughness in heavily drawn pearlitic steels, Materials Letters 58(27-28): 3514-3517.

http://dx.doi.org/10.1016/j.matlet.2004.06.040
Toribio, J.; Valiente, A. 2006. Failure analysis of cold drawn eutectoid steel wires for prestressed concrete, Engineering Failure Analysis 13(3): 301-311.

Kazeem K. ADEWOLE. Dr, holds a Bachelor's and Master's degrees in Civil Engineering and worked for the Standards organization of Nigeria as a Standards Engineer in charge of laboratory testing, standards development and quality assurance of Civil Engineering and Building Materials before going to the United Kingdom for further studies. He completed a Master's degree in Pipeline Engineering from Newcastle University and completed his PhD in the School of Chemical Engineering and Advanced Materials of the University of Newcastle in the United Kingdom. He presently works as a Senior Standards Engineer in the Standards Organization of Nigeria.

Steve J. BULL. Professor. Material Engineer by training, a Professor of Surface Engineering and the immediate past head of the School of Chemical Engineering and Advanced Materials, Newcastle University, United Kingdom. He is also the Cookson Group Chair of Engineering Materials. 